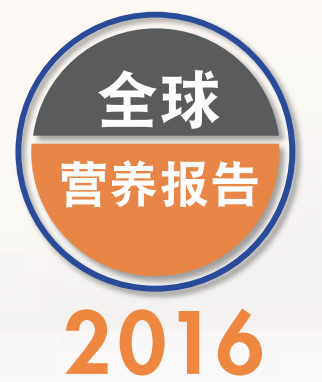

概述

\title{
2030之约: 用行动去影响，消除营养不良
}

在 现今国际社会所面临的诸多挑战中, 营 养不良问题显得尤为严峻：全球有三分之 一的人口营养不良。营养不良有很多不同的表 现形式：儿童生长与发育不良; 个体消瘦或易 受感染; 过度肥胖; 由于过度摄入糖、盐和脂 肪而面临慢性病的风险; 缺乏重要的维生素或 矿物质。就目前来看, 营养不良和不健康饮食 是造成全球疾病负担的最大因素: 各国都面临 着营养不良造成的严峻公共卫生挑战。在非洲 和亚洲, 每年营养不良造成的经济损失相当于 $11 \%$ 的国内生产总值（GDP），而在预防营养 不良方面每投入1美元便可带来16美元的投资回 报。虽然世界各国已就营养目标达成共识, 而 且近年来也取得了一些进展, 但是全球尚未走 上实现这些目标的正轨。这份对世界营养状况 的第三次全面评估为扭转这一趋势并在2030年 前消除一切形式的营养不良指明了方向。

在过去的十年里, 全球在改善营养方面保 持稳步发展势头：2012年，世界卫生大会通过 了“2025全球孕产妇和婴幼儿营养目标”。次 年, 世界卫生大会继续通过了包括营养相关非 传染性疾病在内的非传染性疾病目标。同年, 首届“营养促增长” (N4G) 峰会共筹集230亿美 元承诺捐款用于改善营养行动。随着第二届国 际营养大会(ICN2)于2014年召开以及“20162025联合国营养问题行动十年” 决议于近期通 过, 越来越多的人开始认识到解决一切形式的 营养不良的重要性。2015年, “消除一切形式 的营养不良”目标被郑重纳入联合国可持续发 展目标, 要求全球以不同的方式思考营养不良 问题并采取行动，专注于营养不良问题的各个 方面, 并努力争取到2030年为全人类消除一切 形式的营养不良。

2016年为将这一承诺转化为行动带来了重 大机遇。这些机遇包括：各国通过了与实现可 持续发展目标相关的国家目标; 不断推进和深
化的“营养促增长” 进程; 以及日本为2020年 东京奥运会、残奥会在推动营养安全方面日益 增强的领导作用。

《全球营养报告》是唯一独立而全面的世界 营养状况年度评估报告。它同时也是由众多合 作伙伴协同进行的项目, 反映我们在实现政府 间营养目标方面的成就和不足。该报告还指出 了在全球层面所取得的进展，同时就加快这一 进展给出了建议。《全球营养报告》旨在成为 一座灯塔, 为各国提供成功实例并指明行动的 方向。本年度报告的主题是 “做出并衡量符合 SMART原则的承诺, 制定行动来实现到2030年 消除一切形式的营养不良的目标”。

\section{主要发现}

\section{1 营养不良造成一系列个人和社会问 题, 但同时也带来机遇。}

营养不良和不健康饮食习惯是造成全球 疾病负担的首要因素。我们已经知道, 消瘦、 儿童发育不良以及微营养素缺乏症每年在亚洲 和非洲造成平均 $11 \%$ 的GDP损失, 高于 $2008-$ 2010年金融危机期间的损失。报告提供了有关 营养不良的社会和个人成本的新数据。例如, 在美国, 当家庭中有一人患有肥胖症时, 该家 庭每年需支付的额外医疗费用相当于其年收入 的 $8 \%$ 。在中国, 糖尿病患者每年会因患病损失 $16.3 \%$ 的收入。这些数据意味着, 无论你是否 直接受到营养不良的影响, 营养不良已成为全 人类沉重的负担。但与此同时, 解决营养不良 问题也可以为改善人类福祉和促进经济发展带 来巨大机遇。本报告列举了许多实例来说明一 些国家如何成功利用这些机遇, 通过解决营养 不良问题来改善民生和社会健康。 


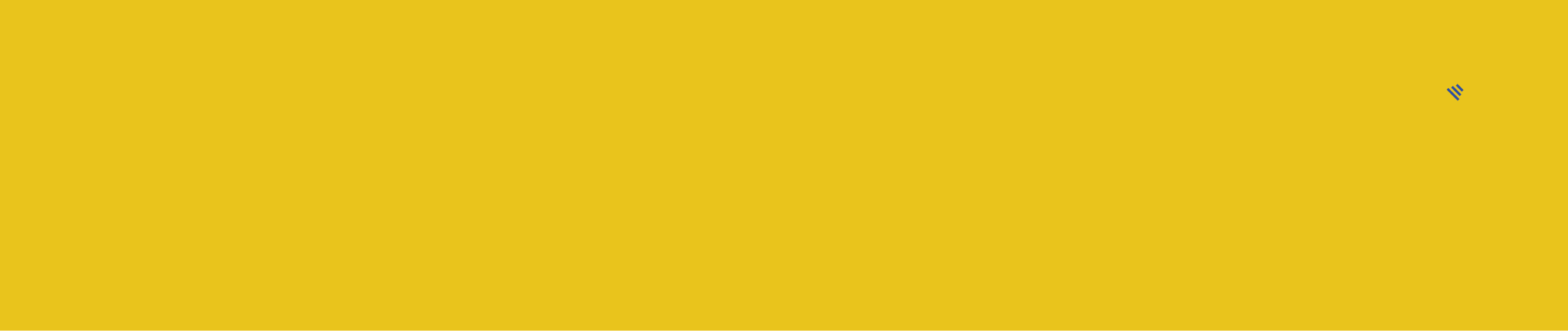

\section{2 全球尚未走上实现营养目标的正 轨, 但希望仍然存在。}

如果我们继续对现状无动于衷, 世界卫生 大会通过的全球营养目标将无法实现。然而, 这一看法掩盖了显著差异和一些惊喜：许多国 家正稳步实现与 5 岁以下儿童发育迟缓、消瘦 和超重以及纯母乳喂养有关的目标。然而, 在 解决女性贫血和成人超重、糖尿病及肥胖症方 面, 几乎所有国家都偏离了轨道。肥胖症和超 重几乎在每个国家每个地区都呈上升趋势, 现 在已成为一项巨大的全球性挑战。5岁以下超 重儿童的数量正逐渐接近消瘦儿童的数量。此 外, 地区性差异不容忽视: 除了非洲和大洋 洲, 每个地区 5 岁以下发育迟缓儿童的数量都在 下降, 而亚洲 5 岁以下超重儿童的数量增加速度 最快。面对这些不容乐观的数据, 我们仍然有 理由怀有希望: 只要稍作调整, 许多国家便可 走上实现全球营养目标的正轨。本报告对这些 机遇进行了概述。

\section{2 营养问题是可持续发展目标的核 心。}

在17个可持续发展目标中, 至少有 12 个 目标包含与营养高度相关的指标, 这反映了营 养在可持续发展中的核心作用。改善营养是在 卫生、教育、就业、女性赋权以及减贫和减少 不平等等方面实现进步的前提。反之, 贫困和 不平等、饮水、环境卫生和个人卫生、教育、 食物系统、气候变化、社会保障和农业均对营 养状况有重要影响。报告表明, 女性权利和地 位和营养状况密切相关：18岁以下的母亲更可 能生育发育迟缓的孩子; 而如果母亲受过中等 教育, 则孩子出现发育迟缓的可能性较低。因 此, 将营养目标纳入发展和社会部门显得尤为 重要, 许多政府在这些领域的支出占总预算的 $30 \%$ 以上。同时, 衡量在这些部门的支出对营 养的影响也十分重要。

\section{当前资金投入无法满足需求。}

鉴于营养不良问题的严重程度, 当前在解 决营养不良问题的资金投入太低。24个中低 等收入国家仅分配 $2.1 \%$ 的支出用于减少营养 不良, 而他们在农业、教育、卫生和社会保障 方面的支出超过 $30 \%$ 。尽管在其他发展和社 会部门, 针对营养不良的措施的资金在增加, 但是用于营养特定干预措施 (nutrition-specific interventions）的资金停滞在10亿美元。营养 相关非传染性疾病（NCDs）方面的投入也明显 不足。目前, 我们还不了解各国分配于应对营 养相关非传染性疾病问题的金额。2014年, 各 国在各种类型的非传染性疾病方面投入的金额 为6.11亿美元, 不足其整体医疗支出的 $2 \%$ 。尽 管, 中低收入国家死亡和残疾人数中有将近一 半是由营养相关非传染性疾病引起的, 但是报 告的新数据显示, 2014年, 捐助者在营养相关 非传染性疾病上分配的金额仅为 5000 万美元。

\section{SMART承诺和目标尤为重要。}

报告发现, 优先考虑营养问题的捐助者和 政府在营养方面支出更多。在营养方面有更多 承诺的企业更有能力 提供能改善营养的产 品、营销策略和标 签。制定营养不良目 标的国家通常也能更 快地减少儿童发育迟 缓。尽管如此, 大 多数营养计划并不 包括完整的全球营
Specific (具体)

Measurable（可衡量）

Achievable（切实可行）

Relevant（相关）

Time bound（有时限性） 养目标, 而且当各

国制定目标时, 仅有三分之二符合SMART的原 则。此外, 仅有30\%的国家在国家层面的非传 染性疾病方案中有减少肥胖症、糖尿病和盐摄 
入量等方面的目标。就 “营养促增长” (N4G)而 言，2013年所作的承诺中仅有29\%符合SMART 原则, 而且大部分承诺未指定他们期望解决哪 些类型的营养不良问题。

\section{我们必须将言语转化为行动。}

报告强调了加强政策与计划实施的必要 性。促进母乳喂养的核心政策和计划严重滞 后：仅有 $36 \%$ 的国家执行了《国际母乳代用品 销售守则》的全部或大部分规定。在向儿童推 销食品和非酒精饮料的监管方面, 所有国家均 未通过全面的方案。三分之二的国家在执行世 界卫生组织促进健康饮食的三大核心建议（减 少盐摄入量、减少反式脂肪与饱和脂肪摄入量 以及执行世卫组织向儿童推销食品和非酒精饮 料的建议) 方面毫无进展。同样, 针对营养不 良的直接方案的推广进程缓慢而不平等。跨部 门行动的协调机制是成功的关键, 但必须为之 提供高层的支持以及人力和财力资源。

\section{7 目前的数据和知识不足以实现投资 的最大化利用。}

报告呼吁营养数据革命。数据的匮乏使 我们无法确定全球和国家层面的真实进展并从 中总结经验。这也掩盖了国家之间的不平等情 况, 致使各国政府更加难以了解实情, 也难以 督促政府全面负责。本报告建议分列数据, 以
便更好地了解存在营养不良的地区：在一项针 对50多个国家的分析中, 发育迟缓率最高的次 国家级地区的发病率是发育迟缓率最低的次国 家级地区的3倍。在13个国家中, 最富有的五分 之一的人群里, 发育迟缓率超过 $20 \%$, 这打破 了高收入必等于营养良好的旧观念。我们面临 严重的数据缺口, 也就是说我们缺乏下列相关 数据：营养敏感型行动以及应对肥胖症和营养 相关非传染性疾病的行动方面的投入; 解决一切 形式的营养不良的方案的覆盖率和影响; 因冲突 而流离失所的 6000 万人口的营养状况; 以及脆 弱国家的营养不良率和趋势。最后, 我们还面临 知识缺口, 让我们难以理解成功或停滞不前的原 因, 以及肥胖症和非传染性疾病的潜在推动因素。

\section{呼吁行动}

\section{1 做出政治选择, 消除一切形式的营 养不良。}

我们尚未走上实现营养目标的正轨。例 如, 贫血症发病率下降速度非常缓慢, 以目前 的速度来看, 我们到 2130 年才能实现这项全球 目标, 而非2030年。肥胖症和超重非但没有下 降, 反而呈上升趋势, 使全球营养安全存在风 险。但是这一不容乐观的局面可以得到改变: 在政府以及做出并遵守承诺的其他组织和个人 的推动下, 巴西、秘鲁、加纳以及印度马哈拉 施特拉邦显著减少了营养不良。从根本上讲,

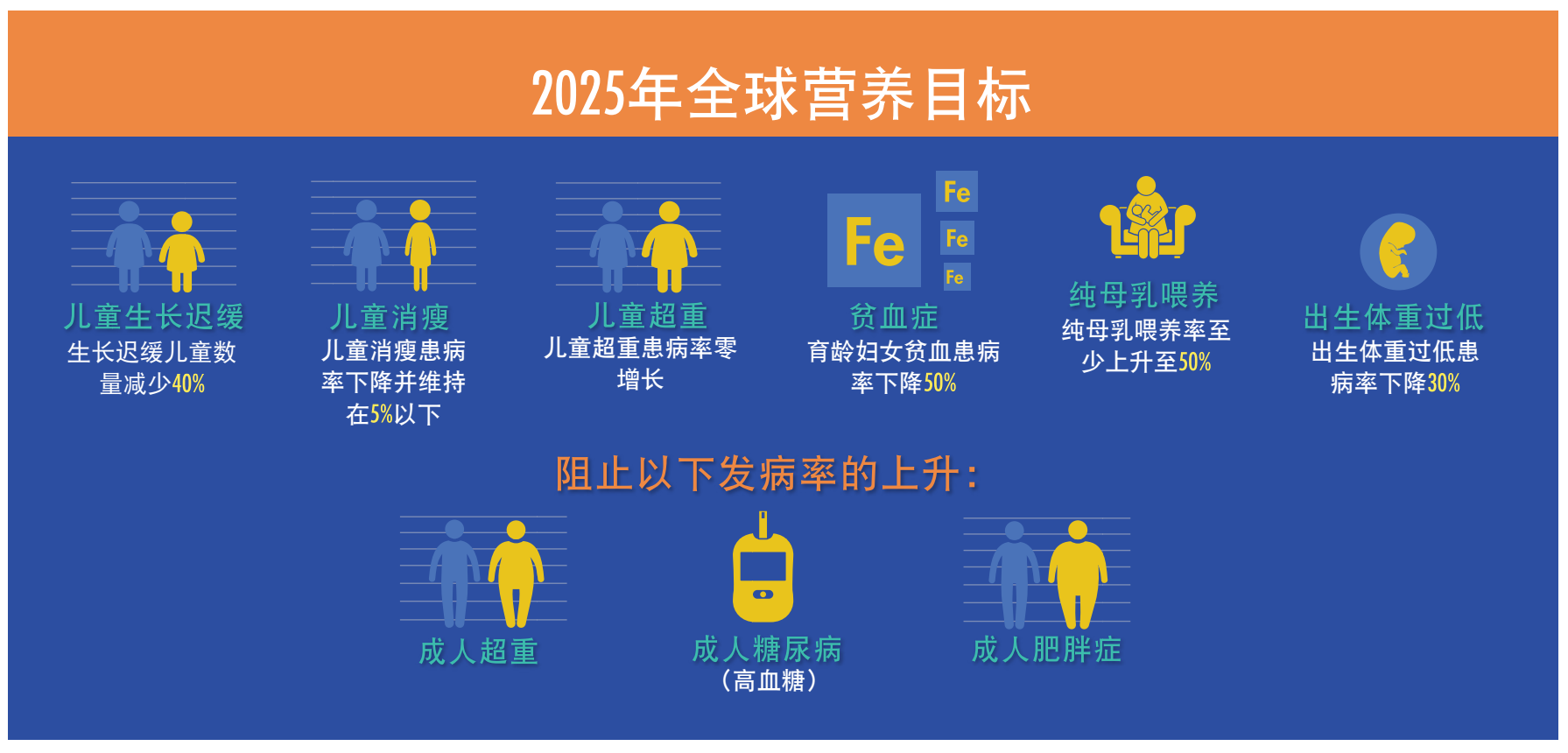


消除营养不良是政府领导人、捐助者、社会团 体以及国际、国内和地区企业需要做出的一个 政治选择。为改善营养做出符合SMART原则的 承诺将帮助世界各国走上正轨。

\section{增加投资、优化分配。}

投资于消除营养不良是各国政府可以采取 的最具成本效益的措施之一：在有效的营养方 案上投资1美元, 便可带来价值16美元的收益。 为了实现重要的全球营养里程碑, 各国政府和 捐助者需要在未来十年将向营养方面的投入提 高到原来的三倍。正如印度马哈拉施特拉邦这 样面临营养不良问题的地区一样，快速增加投 入, 继而改善营养是可以实现的。与此同时, 政府、社会团体、捐助者以及企业需要采取更 多行动, 确保不同部门（农业、教育、食物系 统、卫生系统、社会保障以及饮水、环境卫生 和个人卫生）分配更多资源用于消除一切形式 的营养不良。我们需要更多投入, 以提高解决 肥胖症、糖尿病和其他营养相关非传染性疾病 问题的能力。而且我们需要开始将营养投资视 为促进经济增长的一种手段, 而非将营养改善 视为经济增长的结果。

\section{收集正确的数据以实现投资的最大 化利用。}

数据缺口是全球取得营养进展的一个重 大障碍。每个国家拥有不同的营养状况, 各国 应当收集所需的国家和地区数据, 以便了解自 身独特的营养问题。本着可持续发展目标的精 神, 政府、捐助者、企业和社会团体应当跟踪 并定期报告其在消除一切形式的营养不良（包 括发育迟缓、消瘦、贫血、纯母乳喂养、肥胖 症和非传染性疾病）方面的投入和影响。

\section{在实施有效的解决方案和确定新的 解决方案方面进行投资。}

我们目前拥有足够的经验、数据和证据来 采取行动, 改善营养状况。来自巴西、加纳、
WWW.GLOBALNUTRITIONREPORT.ORG

汶览网站查看:

- 完整的《全球营养报告》

- 193个国家、28个地区和次区域以及全球 的营养概况

- 数据和数据可视化

- 博客

\#NutritionReport @GNRReport

秘鲁等国家的成功实例对其他国家具有指导意 义。我们了解哪些干预措施可以最有效地解决 营养不良问题。我们知道哪些公共政策最有可 能有效减少一切形式的营养不良。我们也已经 认识到，与公民和社会团体携手合作并建立跨 部门的治理机制尤为重要。与此同时, 各国政 府、资助方和研究人员应当携手合作, 努力缩 小阻碍行动的知识缺口：例如, 如果我们缺乏 对消瘦、纯母乳喂养、肥胖症和超重的潜在推 动因素的认识, 我们就不能从卫生部门以外的 部门调动资源来预防。更多地了解一些国家可 以克服障碍并实现高覆盖率的营养计划而另一 些国家却做不到的原因, 将有助于克服瓶颈。 同时确定更具经济效益的新方式, 以便利用现 有的地区数据并收集新数据, 将有助于确保在 可持续发展目标时代各国齐头并进。

\section{5 解决一切形式的营养不良。}

政府、企业、社会团体和个人需要解决一 切形式的营养不良。这意味着中低收入国家政 府必须在肥胖症和营养相关非传染性疾病形势 更加严峻之前竭力大幅降低营养不良。这些国 家必须将糖尿病和肥胖症的预防和控制纳入其 营养方案中并实施可以解决营养不良问题的政 策和干预措施。这意味着经合组织 (OECD) 国 家必须汲取其他国家的成功经验, 改善应对肥 胖症和非传染性疾病的策略。捐助者必须认识 到营养相关非传染性疾病和肥胖症对全球营养 所构成的威胁。这也意味着利益敒关各方需要 确定和实施能同时解决一种以上营养不良问题 的方案，以提高投资和政策的效率。同时，利 益敒关各方需要携手并进、积极应对解决一切 形式的营养不良问题的 “新常态” , 因为这是 全球近半数国家所面临的一个共同挑战。 